\title{
IMPLICATIONS OF THE SNO AND THE HOMESTAKE RESULTS FOR THE BOREXINO EXPERIMENT
}

\author{
S. M. Bilenky, I T. Lachenmaier, W. Potzel and F. von Feilitzsch \\ Physik-Department, Technische Universität München, \\ James-Franck-Straße, D-85748 Garching, Germany
}

\begin{abstract}
Using the recent result of the SNO solar neutrino experiment, we have demonstrated in a model independent way that the contribution of ${ }^{7} \mathrm{Be}$ and other medium energy neutrinos to the event rate of the Homestake experiment is $4 \sigma$ smaller than the BP2000 SSM prediction. We have considered the implications of this result for the future BOREXINO experiment.
\end{abstract}

The recently published first data of the SNO experiment [1] combined with the data of the Super-Kamiokande solar neutrino experiment [2] present clear evidence of the presence of $\nu_{\mu}$ and/or $\nu_{\tau}$ in the flux of high energy solar neutrinos on the Earth. The large up-down asymmetry of high energy atmospheric muon events, discovered by the Super-Kamiokande collaboration [3], constitutes a model independent evidence in favor of oscillations of atmospheric neutrinos. The data of all solar and atmospheric neutrino experiments can be described by practically decoupled two-neutrino oscillations driven by the mixing of three neutrinos with neutrino mass squared differences that satisfy the hierarchy $\Delta m_{\text {sol }}^{2} \ll \Delta m_{\text {atm }}^{2}$ (see 四).

There exist at present indications in favor of $\bar{\nu}_{e} \rightarrow \bar{\nu}_{\mu}$ oscillations that were obtained in the accelerator experiment LSND [5]. If the data of the LSND experiment will be confirmed, we must assume that (at least) four mixed neutrinos with small masses exist in nature and that flavor neutrinos can transfer into sterile states. A check of the LSND result is going to be achieved by the MiniBooNE experiment [6], scheduled to start in 2002.

The SNO result triggered several new global analysis of the data of all solar neutrino experiments [7, 8] . After the Super-Kamiokande measurement [2] of the energy spectrum of recoil electrons and the zenith angle dependence of the flux of solar neutrinos large mixing angle MSW solutions became the most favorable fits [9] of all pre-SNO solar neutrino data ([2, 10, 11, 12, 13]).

\footnotetext{
${ }^{1}$ Also at Joint Institute for Nuclear Research, Dubna, Russia.
} 
The inclusion of the SNO data in the global analysis only strengthens this conclusion [7, 8].

The result of the SNO experiment together with results of all other solar neutrino experiments allowed to obtain the average values of the $\nu_{e}$ survival probability in the high energy, medium energy and low energy regions [14, 15]. In [15 the general case of transitions of the solar $\nu_{e}$ 's into flavor and sterile neutrinos was considered. In [14] the averaged values of the survival probability were compared with the survival probability that can be inferred from global analysis of the solar neutrino data. In [16] a new phenomenological analysis of all solar neutrino data, including SNO data, was done. It was assumed that there are no transitions of solar $\nu_{e}$ 's into other states and that total solar fluxes are free fit parameters. A 7.4 $\sigma$ disagreement between the measured and the fitted values of the total event rates was found.

The Super-Kamiokande and SNO solar neutrino experiments provide evidence of oscillations of the high energy ${ }^{8} \mathrm{~B}$ solar neutrinos. The next solar neutrino experiment BOREXINO [17], scheduled for 2002, is aimed to detect medium energy ${ }^{7} \mathrm{Be}, \mathrm{CNO}$ and pep neutrinos. In this paper we will infer possible implications for the BOREXINO experiment from the results of the SNO and the Homestake solar neutrino experiments.

The Homestake experiment is sensitive mainly to neutrinos from ${ }^{8} \mathrm{~B}$ decay and ${ }^{7} \mathrm{Be}$ capture. According to the SSM BP2000 [18] neutrinos from ${ }^{15} \mathrm{O}$ and ${ }^{13} \mathrm{~N}$ decays and the pep reaction give $\simeq 8.4 \%$ of the total contribution to the chlorine event rate.

The event rate in a chlorine experiment is given by

$$
R_{C l}=\int_{E_{t h}} \sigma_{\nu_{e} C l}(E) \sum_{i} \Phi_{\nu_{e}}^{i}(E) d E .
$$

Here $\sigma_{\nu_{e} C l}$ is the cross section of the process

$$
\nu_{e}+{ }^{37} \mathrm{Cl} \rightarrow \mathrm{e}^{-}+{ }^{37} \mathrm{Ar},
$$

$\Phi_{\nu_{e}}^{i}(E)$ is the flux of the solar $\nu_{e}$ 's from the source $i$ on the Earth $\left(\mathrm{i}={ }^{8} \mathrm{~B}\right.$, ${ }^{7} \mathrm{Be},{ }^{15} \mathrm{O},{ }^{13} \mathrm{~N}$, pep) ${ }^{2}$ and $E_{t h}=0.814 \mathrm{MeV}$ is the threshold of the process (2). We have

$$
\Phi_{\nu_{e}}^{i}(E)=P\left(\nu_{e} \rightarrow \nu_{e}\right) \Phi_{\nu_{e}}^{i 0}(E)
$$

where $P\left(\nu_{e} \rightarrow \nu_{e}\right)$ is the $\nu_{e}$ survival probability, $E$ is the neutrino energy and $\Phi_{\nu_{e}}^{i 0}$ is the initial flux of electron neutrinos from the source $i$.

\footnotetext{
${ }^{2}$ We will not consider the small contribution of the hep neutrinos.
} 
Using now the results of the SNO experiment we will determine the contribution of the ${ }^{8} \mathrm{~B}$ neutrinos to the chlorine event rate $R_{C l}$.

In the SNO experiment solar $\nu_{e}$ 's are detected through the observation of the charged current $(\mathrm{CC})$ process

$$
\nu_{e}+d \rightarrow e^{-}+p+p
$$

The neutrino energy threshold in the SNO experiment is equal to $E_{t h}=$ $T_{t h}+1.44 \mathrm{MeV}=8.19 \mathrm{MeV}$ where $T_{t h}=6.75 \mathrm{MeV}$ is the electron kinetic energy threshold. For the observed event rate we have [19]

$$
R_{S N O}=\left\langle\sigma_{\nu_{e} d}\right\rangle \Phi_{\nu_{e}}^{S N O}
$$

Here $\left\langle\sigma_{\nu_{e} d}\right\rangle$ is the cross section of the process (4) averaged over the initial spectrum of the ${ }^{8} \mathrm{~B}$ neutrinos and

$$
\Phi_{\nu_{e}}^{S N O}=\left\langle P\left(\nu_{e} \rightarrow \nu_{e}\right)\right\rangle_{C C} \Phi_{\nu_{e}}^{8 B 0}
$$

is the flux of $\nu_{e}$ on the Earth and $\Phi_{\nu_{e}}^{8 B}$ is the total initial flux of the ${ }^{8} \mathrm{~B}$ neutrinos.

In the SNO experiment[1] it was found that

$$
\left.\Phi_{\nu_{e}}^{S N O}=(1.75 \pm 0.07 \text { (stat.) } \pm 0.12 \text { (sys.) } \pm 0.05 \text { (theor. })\right) \cdot 10^{6} \mathrm{~cm}^{-2} \mathrm{~s}^{-1}
$$

No statistically significant distortion of the energy spectrum of the recoil electrons in the region $5-20 \mathrm{MeV}$ with respect to the spectrum, predicted under the hypothesis of a constant survival probability, was observed in the Super-Kamiokande experiment [2]. No distortion of the electron spectrum was observed also in the SNO experiment in the energy range 8.2 - 14.4 $\mathrm{MeV}$. Thus, data of the SNO and the Super-Kamiokande experiments are in agreement with the assumption of a constant $\nu_{e}$ survival probability in the high energy region.

We will assume first that the $\nu_{e}$ survival probability for ${ }^{8} \mathrm{~B}$ neutrinos is constant in the whole energy range of the Homestake experiment. Then we will calculate the corrections due to the possible dependence of the survival probability on the neutrino energy in the energy range from the threshold of the Homestake experiment to the threshold of the SNO experiment.

If we assume a constant survival probability in the whole Homestake energy range we can calculate the contribution of the ${ }^{8} \mathrm{~B}$ neutrinos to the chlorine event rate using directly the SNO result. We have 


$$
\left(R_{C l}^{8}\right)_{S N O}=\int_{E_{t h}} \sigma_{\nu_{e} C l}(E) X^{8} B(E) \Phi_{\nu_{e}}^{S N O} d E=(2.00 \pm 0.17) \mathrm{SNU}
$$

Here $X^{8} B(E)$ is the (known) normalized spectrum of the initial ${ }^{8} \mathrm{~B}$ neutrinos $\left(\int X^{8} B(E) d E=1\right)$. The flux of the ${ }^{8} \mathrm{~B}$ neutrinos on the Earth $\Phi_{\nu_{e}}^{S N O}$, measured in the SNO experiment, is given by (7). The uncertainty in (8) is determined by the uncertainties of the SNO flux and of the cross section of the process (2).

Let us now estimate the possible effect of the energy dependence of the probability $P\left(\nu_{e} \rightarrow \nu_{e}\right)$ in the energy range from the Homestake threshold $\left(E_{t h} \simeq 0.81 \mathrm{MeV}\right)$ to the SNO threshold $\left(E_{t h} \simeq 8.19 \mathrm{MeV}\right)$. The contribution of the ${ }^{8} \mathrm{~B}$ neutrinos to the chlorine event rate $R_{C l}^{8} B$ can be presented in the form

$$
R_{C l}^{8 B}=\left(R_{C l}^{8 B}\right)_{S N O}+Q
$$

where the quantity $Q$ is given by

$$
Q=\int_{0.81 \mathrm{MeV}}^{8.19 \mathrm{MeV}} \sigma_{\nu_{e} C l}(E) X^{8} B(E)\left(P\left(\nu_{e} \rightarrow \nu_{e}\right) \Phi_{\nu_{e}}^{8} B^{B 0}-\Phi_{\nu_{e}}^{S N O}\right) d E
$$

The total flux of the active ${ }^{8} \mathrm{~B}$ neutrinos on the Earth, that can be inferred from the results of the SNO and the Super-Kamiokande experiments, is in good agreement with the value predicted by the BP2000 SSM. Thus, we will assume that the flux $\Phi_{\nu_{e}}^{8} B_{0}$ in Eq.(10) is given by the SSM. For the survival probability $P\left(\nu_{e} \rightarrow \nu_{e}\right)$ we used the expressions that can be obtained from the global analysis of the solar neutrino data [20]. We have found that for the preferable large mixing angle MSW solutions the quantity $Q$ is significantly smaller than the error in (8): $Q \simeq-0.05 \mathrm{SNU}$ for the LMA solution and $Q \simeq 0.08 \mathrm{SNU}$ for the LOW solution. Thus, we can neglect $Q$ in (9) and for the contribution of the ${ }^{8} \mathrm{~B}$ neutrinos to the chlorine event rate we can take the value (8).

The event rate, measured in the Homestake experiment [10], is equal to

$$
R_{C l}=(2.56 \pm 0.16 \pm 0.16) \quad \mathrm{SNU}
$$

If we subtract (8) from (11) we will find a model independent contribution of ${ }^{7} \mathrm{Be}, \mathrm{CNO}$ and pep neutrinos to the Homestake event rate ${ }^{3}$

\footnotetext{
${ }^{3}$ The errors were added in quadrature.
} 


$$
R_{C l}^{7 B e, C N O, p e p}=(0.56 \pm 0.29) \mathrm{SNU}
$$

According to the BP2000 SSM [18], the contribution of ${ }^{7} \mathrm{Be}$ neutrinos to the chlorine event rate is equal to $1.15 \mathrm{SNU}$ and the total contribution of the medium energy neutrinos is equal to $1.79 \mathrm{SNU}$. Thus the value (12) constitutes a model independent $4 \sigma$ evidence, of the solar neutrino problem for ${ }^{7} \mathrm{Be}$ and other medium energy neutrinos based on the comparison of the SNO and Homestake results.

Let us now compare the rate (12) with the preferable global fits [9, 7 , 8, 20] of the solar neutrino data. In the scenario of the LMA solution the ${ }^{7} \mathrm{Be}$ contribution to the chlorine rate is equal to $0.53 \mathrm{SNU}$ and the total contribution of the medium energy neutrinos is equal to $0.77 \mathrm{SNU}$. In the case of the LOW solution the ${ }^{7} \mathrm{Be}$ contribution to the chlorine rate is equal to $0.55 \mathrm{SNU}$ and the total contribution is equal to $0.83 \mathrm{SNU}$.

Let us stress that the value (12) was obtained directly from the results of the SNO and the Homestake experiments. No assumptions on the fluxes of ${ }^{7} \mathrm{Be}, \mathrm{CNO}$ and pep neutrinos and $\nu_{e}$ survival probabilities were made. On the other side, the global analysis of the solar neutrino data is based on the assumption of two-neutrino oscillations and on SSM fluxes. In spite of the large error in (12) the fact that (12) is compatible with the contribution of the medium energy neutrinos to the $\mathrm{Cl}$ event rate, predicted by the LMA and LOW solutions, from our point of view, is a model independent argument in favor of these solutions.

The SMA MSW solution for the contribution of the medium energy neutrinos to the chlorine event rate predicts the value $0.01 \mathrm{SNU}$, which is in $2 \sigma$ disagreement with (12). This illustrates the preference of the large mixing angle MSW solutions, that follows from the direct comparison of the Homestake and SNO results.

Taking into account that (12) is the contribution of all medium energy neutrinos to the chlorine event rate for the contribution of the prevailing $0.862 \mathrm{MeV}^{7} \mathrm{Be}$ neutrinos we can obtain only an upper bound. From (12) we have for the ${ }^{7} \mathrm{Be}$ contribution to the chlorine rate at a $1 \sigma$ level

$$
R_{C l}^{7 B e} \leq 0.85 \mathrm{SNU}
$$

This gives a model independent upper bound for the flux of the ${ }^{7} \mathrm{Be}$ neutrinos

$$
\Phi_{\nu_{e}}^{7} \mathrm{Be} \leq 3.16 \cdot 10^{9} \mathrm{~cm}^{-2} \mathrm{~s}^{-1},
$$


This upper bound comprises $\leq 74 \%$ of the BP2000 SSM flux of the 0.862 $\mathrm{MeV}{ }^{7}$ Be neutrinos.

In order to obtain from (12) the value of the flux of the ${ }^{7} \mathrm{Be}$ neutrinos we will assume that the contributions of CNO and pep neutrinos to the chlorine event rate $R_{C l}^{C N O, p e p}$ are given by the LMA and LOW MSW solutions. We find [20]

$$
R_{C l}^{C N O, p e p}=0.24 \mathrm{SNU}(\mathrm{LMA}) ; \mathrm{R}_{\mathrm{Cl}}^{\mathrm{CNO}, \mathrm{pep}}=0.29 \mathrm{SNU}(\mathrm{LOW})
$$

From (12) and (15) we have for the contribution of the ${ }^{7}$ Be neutrinos to the chlorine event rate

$$
R_{C l}^{7} B e=0.32 \pm 0.29 \text { SNU }(\mathrm{LMA})
$$

and

$$
R_{C l}^{7} B e=0.27 \pm 0.29 \mathrm{SNU}(\mathrm{LOW})
$$

Let us stress that in (16) and (17) we do not include the $\simeq 20 \%$ estimated theoretical error [18] of the SSM CNO flux.

For the flux of the ${ }^{7} \mathrm{Be}$ neutrinos on the Earth we have from (16) and (17), respectively

$$
\Phi_{\nu_{e}}^{7 B e}=(1.19 \pm 1.08) \cdot 10^{9} \mathrm{~cm}^{-2} \mathrm{~s}^{-1}
$$

and

$$
\Phi_{\nu_{e}}^{7} B e=(1.00 \pm 1.08) \cdot 10^{9} \mathrm{~cm}^{-2} \mathrm{~s}^{-1}
$$

The fluxes (18) and (19) are about $3 \sigma$ smaller than the flux of the ${ }^{7} \mathrm{Be}$ neutrinos $4.28(1.00 \pm 0.10) \cdot 10^{9} \mathrm{~cm}^{-2} \mathrm{~s}^{-1}$, predicted by BP2000 SSM 18.

The flux $\Phi_{\nu_{e}}^{7 B e}$ is the product of the initial flux of the ${ }^{7} B e$ neutrinos $\Phi_{\nu_{e}}^{7} B e 0$ and the probability of the ${ }^{7} \mathrm{Be}$ neutrinos to survive. If for the flux $\Phi_{\nu_{e}}^{7_{e}}{ }^{\nu_{e}}$ we take the SSM value, for the survival probability we find from (18) and(19) respectively

$$
P^{7} B e\left(\nu_{e} \rightarrow \nu_{e}\right)=0.28 \pm 0.25
$$

and

$$
P^{7} B e\left(\nu_{e} \rightarrow \nu_{e}\right)=0.23 \pm 0.25
$$

Finally we will present the rates for the future BOREXINO experiment [17], expected from the phenomenological analysis of the results of the SNO and Homestake experiments given above. In the liquid scintillator experiment BOREXINO the medium energy ${ }^{7} \mathrm{Be}, \mathrm{CNO}$ and pep neutrinos will be 
detected via the observation of recoil electrons from elastic neutrino-electron scattering

$$
\nu+e \rightarrow \nu+e
$$

The energy window of the experiment is $250-800 \mathrm{keV}$. In the calculation of the major contribution of the ${ }^{7} \mathrm{Be}$ neutrinos to the event rate we used the fluxes given by (18) and (19). In the calculation of the corrections due to CNO and pep neutrinos we used the LMA and LOW solutions. For the expected total event rates in the BOREXINO experiment we found, correspondingly

$$
R_{\text {BOREX }}=24.4 \pm 8.6 \text { events } / \text { day } ; R_{\text {BOREX }}=22.8 \pm 8.6 \text { events } / \text { day }
$$

The dominant contribution to the errors in (23) comes from the error of the quantity $R_{C l}^{7} B e, C N O$,pep given by (12).

It is interesting to compare the rates (23) with the rates predicted by the favorable large mixing angle MSW solutions. The contribution of ${ }^{7} \mathrm{Be}$ neutrinos to the BOREXINO event rate is equal to 24.4 events/day for the LMA solution and 22.8 events/day for the LOW solution. The total contribution to the BOREXINO event rate of neutrinos from all sources is equal to 30.7 events/day for the LMA solution and 29.0 events/day for the LOW solution. Thus, taking into account the errors in (23), the total BOREXINO event rate, predicted directly from the results of the SNO and the Homestake experiments (for the dominant ${ }^{7} \mathrm{Be}$ neutrinos), is compatible with the rates predicted by the favorable solutions of the solar neutrino problem.

Notice that the SMA solution for the BOREXINO event rate predicts 11.7 events/day, about $1.5 \sigma$ smaller than (22) and BP2000 SSM predicts 55.2 events/day, about $3.6 \sigma$ larger than (23).

In conclusion, from the recent result of the SNO experiment [1] and the result of the Homestake experiment [10] we have obtained the contribution of ${ }^{7} \mathrm{Be}$ and other medium energy neutrinos to the chlorine event rate. We have shown that this contribution is about $4 \sigma$ smaller than the value predicted by BP2000 SSM. The flux of ${ }^{7}$ Be neutrinos we have obtained directly from experimental data in a model independent way. In the calculations of the corrections due to CNO and pep neutrinos we used the large mixing angle MSW solutions that after recent Super-Kamiokande [2] and SNO [1] measurements became the preferred solutions of the solar neutrino problem. Using the flux of ${ }^{7} \mathrm{Be}$ neutrinos, that was inferred from the SNO and Homestake results, we have calculated the expected event rate for the future BOREXINO experiment. 
This work has been supported by SFB 375 für Astroteilchenphysik der

DFG. S. Bilenky acknowledges the Alexander von Humboldt Foundation for support.

\section{References}

[1] SNO collaboration, Q.R. Ahmad et al. Phys. Rev. Lett. 87, 071301 (2001).

[2] Super-Kamiokande Collaboration, S. Fukuda et al. Phys. Rev. Lett. 86, 5651 (2001).

[3] Super-Kamiokande Collaboration, S. Fukuda et al. Phys. Rev. Lett. 81, 1562 (1998), S. Fukuda et al. Phys. Rev. Lett. 82, 2644 (1999), S. Fukuda et al. Phys.Rev.Lett.85, 3999 (2000).

[4] S.M. Bilenky, C. Giunti and W. Grimus, Prog. Part. Nucl. Phys. 43, 1 (1999), hep-ph/9812360.

[5] LSND Collaboration, talk by G. Mills, Proceedings of the 19th International Conference on Neutrino Physics and Astrophysics, Neutrino 2000 (Sudbury, Canada, June 16-21, 2000).

[6] MiniBooNE Collaboration, talk by A. Bazarko, Proceedings of the 19th International Conference on Neutrino Physics and Astrophysics, Neutrino 2000 (Sudbury, Canada, June 16-21, 2000).

[7] J. N. Bahcall, M. C. Gonzalez-Carcia, C.Pena-Garay, hep-ph/0106258.

[8] G. L. Fogli, E. Lisi, D. Montanino and A. Palazzo, hep-ph/0106247.

[9] S. Fukuda et al., Phys. Rev. Lett. 86, 5656 (2001).

[10] B. T. Cleveland et al., Astrophys. J. 496, 505 (1998).

[11] Kamiokande Collaboration, Y. Fukuda et al., Phys. Rev. Lett. 77, 1683 (1996).

[12] SAGE Collaboration, J. N. Abdurashitov et al., Phys. Rev. C 60, 055801 (1999). 
[13] GALLEX-GNO Collaboration, M. Altmann et al., Phys. Lett. B 490, 16 (2000); GALLEX Collaboration, W. Hampel et al. Phys. Lett. B 447, 127 (1999).

[14] V. Berezinsky, M.Lissia, hep-ph/0108108.

[15] V. Barger, D. Marfatia and K.Whisnant, hep-ph/0106207.

[16] J. N. Bahcall, hep-ph/0108147.

[17] BOREXINO Collaboration, talk by G. Ranucci, Proceedings of the 19th International Conference on Neutrino Physics and Astrophysics, Neutrino 2000 (Sudbury, Canada, June 16-21, 2000).

[18] J. N. Bahcall, M. H. Pinsonneault and S. Basu, Astrophys. J. 555, 990 (2001).

[19] F. L. Villante, G. Fiorentini, and E. Lisi, Phys. Rev. D 59, 013006 (1999).

[20] J. N. Bahcall, P.Krastev and A.Smirnov, Phys. Rev. D 58, 096016 (1998). 